\title{
Prioritising downgaze alignment in the management of vertical strabismus for thyroid eye disease: principles and outcomes
}

\author{
J. M. Jefferis ${ }^{1} \cdot$ N. Raoof ${ }^{2,3} \cdot$ J. P. Burke ${ }^{1}$
}

Received: 7 February 2019 / Revised: 7 August 2019 / Accepted: 12 August 2019 / Published online: 30 September 2019

(c) The Author(s), under exclusive licence to The Royal College of Ophthalmologists 2019

\begin{abstract}
Objective To evaluate outcomes of surgery for vertical strabismus in thyroid eye disease (TED) employing a set of surgical principles aimed at avoiding reversal of downgaze deviation while restoring binocular single vision (BSV).

Methods A retrospective review of consecutive patients undergoing vertical strabismus surgery for TED using a set of surgical principles between 2008 and 2017. Principle outcome measure was the presence of BSV in primary position and downgaze 3 months postoperatively and at latest follow-up.

Results Thirty five patients (29\% male) with a mean age of 58 years (range 31-83 years) were included. Median follow-up was 16 months. At presentation, 17 (49\%) used monocular occlusion to avoid diplopia; the remainder used a prism and/or abnormal head posture. In 12 (34\%), combined horizontal and vertical muscle surgery was required. Median (inter-quartile range) preoperative vertical deviations in primary position and downgaze respectively were 20 prism dioptres $(\Delta)(15,30)$ and $18 \Delta(8,22)$, which improved to $1 \Delta(0,3)$ and $1 \Delta(0,3)$ after the first operation. BSV in primary position and downgaze, without prism, after a single surgery was achieved in $29(83 \%)$. Second surgery was required in $5(14 \%)$ and one patient who had reactivation of her TED required a third surgery. At final follow-up (median 16 months), 32 (91\%) were diplopia free

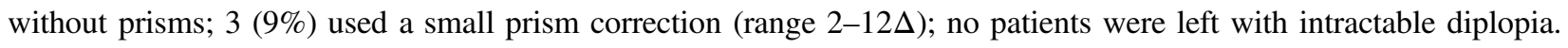

Conclusions Our management principles are characterised by prioritising downgaze alignment to avoid downgaze diplopia reversal, whilst limiting adjustable sutures and employing standard surgical dosing. They give favourable outcomes in TED associated vertical strabismus.
\end{abstract}

\section{Introduction}

Symptoms of disturbed extraocular motility are seen in $15 \%$ of patients with thyroid eye disease (TED) [1]. Diplopia onset is typically insidious, although it may be sub-acute, and whilst not widely documented in clinical research studies, it is recognised in the clinical setting to be associated with a spectrum of vertical fusion amplitudes, from

$\square$ J. M. Jefferis

jojefferis@ doctors.org.uk

1 Department of Ophthalmology, Royal Hallamshire Hospital, Sheffield, UK

2 Moorfields Eye Hospital NHS Trust, City Road, London EC1V 2PD, UK

3 Department of Ophthalmology, Royal London Hospital, Barts Health NHS Trust, Whitechapel Road, Whitechapel, London E1 $1 \mathrm{BB}, \mathrm{UK}$ ineffectual to much enlarged [2,3]. Its incomitant, restrictive nature poses a management challenge that is reflected in the relatively high rate of re-operation (4-45\%) described in the literature [4]. For some patients re-operations will be planned or anticipated, particularly in those with complex combined vertical and horizontal strabismus requiring uni/ bilateral multi-muscle surgery ( $>2$ muscles per eye).

The aim of strabismus surgery is to restore binocular single vision (BSV) in primary position and reading position with as large a field of BSV as practically possible [5]. The most common muscles affected are the inferior and medial recti, which are managed with varying amounts of recession [6, 7]. Whilst there may be a role for standardised dosing tables [8], these are generally considered insufficient alone in surgical planning [6,9]. Several ancillary techniques have been described where, rather than calculating surgical dosing based on angles of deviation, surgical management involves assessment of forced duction tests (FDTs) and recessing restricted muscles. Nguyen et al. describe a method of correction of duction deficits which 
they found favourable [10]. However, others have failed to replicate Nguyen's results [11]. A similar method to the correction of duction deficits, is the intraoperative relaxed muscle technique $[9,12]$. A number of surgeons have advocated the use of adjustable sutures, most commonly on the recessed inferior rectus [13-15]. However, even with adjustable suture techniques, up to $33 \%$ of patients may still need prisms postoperatively and $17 \%$ re-operation $[13,16]$.

Several symptom producing surgical complications that can be difficult to manage need avoiding. Reversal of vertical deviation in downgaze gives unfavourable outcomes [17]. Large inferior rectus recessions can induce an A-pattern; especially if performed in conjunction with medial rectus recession [14, 18]. Some reports indicate that this can be ameliorated by medial transposition of the recessed inferior rectus but this can then give problematic incyclotorsion $[4,6]$.

In this study, we describe our experience of managing vertical diplopia by employing an alogrithm that prioritises elimination of symptoms in downgaze. In surgical planning, we pay particular attention to the vertical deviation in downgaze; the size of any pre-existing vertical fusion range; magnitude (in prism dioptres) of vertical disparity between downgaze and primary position; and intraoperative FDTs. Our surgery aims primarily to avoid reversal of hypotropia in all but extreme downgaze with balanced ductions/versions in downgaze; this may be at the expense of persistent (albeit reduced) diplopia and asymmetry of ductions in elevation.

\section{Subjects and methods}

Approval for this study of our established surgical practice was granted in line with protocols at Sheffield Teaching Hospitals NHS Trust; local ethics committee approval was deemed not required. We retrospectively reviewed medical records of consecutive patients with TED having strabismus surgery for vertical misalignment. All surgery was carried out at Sheffield teaching hospitals, UK, by a single surgeon (JB) between January 2008 and December 2017. Patients were excluded if they had had previous strabismus surgery in another eye unit or if they had a $\log$ MAR visual acuity worse than 0.6 (6/24 Snellen equivalent) in either eye

The diagnosis of TED was made by history, clinical examination and investigation findings (thyroid autoantibody and thyroid biochemistry studies, computed tomography and/or magnetic resonance imaging). Assessment consisted of best corrected visual acuity, slit lamp biomicroscopy, fundus examination, tonometry and serial motility examinations by an orthoptist showing stability before surgery. The operating surgeon remeasured all patients preoperatively. Postoperatively, measurements were taken by an orthoptist (not masked) 1 day postoperatively, 2 weeks postoperatively, 3 months postoperatively and at latest follow-up. Restricted ductions were graded on an ordinal scale from 0 (normal) to -6 (cannot rotate eye to within $50 \%$ of midline). Ocular deviations were measured using prism cover tests at $1 / 3 \mathrm{~m}$ and at $6 \mathrm{~m}$ in nine positions of gaze in prism dioptres $(\Delta)$. Cyclotorsion was measured with a synoptophore and where appropriate examination for fundal torsion. To determine the magnitude of vertical motor fusion, first the deviation was neutralised in either primary or downgaze to achieve BSV, then the prism fusion range measured in the direction of vertical misalignment. Alternatively, the largest vertical phoria whilst using an abnormal head posture was measured. The field of BSV was measured in free space estimating the extent of BSV in up, down, left and right gaze. A standard orthopaedic goniometer (Patterson medical) was used to measure (in degrees) extent of upward and downward gaze, as well as abnormal head posture, where applicable. 'Functional downgaze' describes the qualitative, individual specific area that extends below the midline where any vertical phoria must be compatible with the maintenance of comfortable BSV while performing one's daily living activities. Quantitatively this can be expected to correlate with the absence of iatrogenic vertical alignment reversal or residual vertical tropia at $30-35^{\circ}$ into depression.

\section{Surgical principles and procedure}

Informed consent was obtained from all patients. Surgery was carried out under general anaesthesia. Patients had peroperative FDTs. Limbal incisions were used. Muscles were recessed from the original muscle insertion and all measurements taken from the limbus. Absorbable 6:0 vicryl sutures were used for the muscles, except in a minority of cases with very tight inferior recti where non-absorbable 5:0 dacron was used.

The following principles were used to plan surgery; principles 2 and 3 are uniquely relevant with respect to the target goal and focus on downgaze alignment.

1. Choice of muscles for surgery was made following preoperative motility examination and per-operative FDTs, with the aim of:
a. Achieving downgaze duction symmetry
b. Reducing (but not necessarily relieving or sym- metrising) restricted upgaze ductions

2. If the patient had an enlarged vertical fusion reserve, this was utilised to ameliorate any incomitance 
between primary position and downgaze (see Fig. 2)

3. Where there was significant incomitance between primary position and downgaze but no enlarged fusional reserve, the angle of deviation in functional downgaze was used as a target angle of correction (see Fig. 3)

4. Standardised surgical dosing was used, where $1 \mathrm{~mm}$ of vertical muscle recession equates to $2.5-3.0$ prism dioptres of correction.

5. Where excyclotorsion was a barrier to achieving preoperative BSV, unilateral or bilateral inferior rectus recessions were used to eliminate the excyclotorting effect of tight inferior recti.

6. Excessive simultaneous bilateral inferior recti recessions ( $>12.5 \mathrm{~mm}$ from the limbus bilaterally) were avoided in order to reduce the likelihood of inducing A patterns.

7. Adjustable sutures were reserved for cases with complex restrictive disease, or small vertical prism fusion ranges and were usually placed on a recessed superior rectus. Adjustments were made on the day of surgery with the immediate postoperative adjustment goal being to avoid any overcorrection or reversal of vertical deviation in anything but extreme (c.50 $)$ downgaze, with small undercorrection in primary position.

8. Simultaneous horizontal muscle surgery was undertaken as appropriate to facilitate BSV postoperatively based on magnitude of horizontal and vertical misalignment and motor fusion amplitudes.

9. All operated muscles were recessed and no more than two recti per eye were operated in a single surgery.

\section{Outcome measures}

Our primary outcome measure was the presence of BSV in primary position and functional downgaze 3 months postoperatively (Fig. 1), as well as at final follow-up. Other outcome measures were magnitude of the disparity between the deviation in primary position and downgaze (in prism dioptres), need for re-operation, and use/size of prism correction at final follow-up.

\section{Statistical analysis}

Statistical analysis was carried out using IBM SPSS statistics (version 25). Kurtosis $z$-scores were used to determine if data approximated a normal distribution. Data approximating a normal distribution was described using means and standard deviations, whereas non-normal data were described using medians and inter-quartile ranges or ranges.
Table 1 Baseline characteristics of the 35 patients included in the study

\begin{tabular}{lc}
\hline Gender: & \\
$\quad$ Male, $n(\%)$ & $10(29 \%)$ \\
$\quad$ Female, $n(\%)$ & $25(71 \%)$ \\
Age at time of first strabismus surgery, mean (std) & $58.3(12.9)$ \\
Current or recent ex-smoker, $n(\%)$ & $20(57.1 \%)$ \\
Previous treatment for TED: & \\
$\quad$ Steroid sparing immunosuppression, $n(\%)$ & $7(20.0 \%)$ \\
High dose steroid, $n(\%)$ & $20(57.1 \%)$ \\
$\quad$ Orbital radiotherapy, $n(\%)$ & $10(28.6 \%)$ \\
$\quad$ Orbital decompression surgery, $n(\%)$ & $16(45.7 \%)$ \\
LogMAR best corrected vision & \\
$\quad$ Better seeing eye, mean (std) & $0.07(0.10)$ \\
Poorer seeing eye, mean (std) & $0.19(0.16)$
\end{tabular}

$T E D$ thyroid eye disease, $S T D$ standard deviation

\section{Results}

A total of 39 patients had vertical strabismus surgery for TED during the time period examined, three patients were excluded due to a history of previous strabismus surgery elsewhere. A further one patient was excluded due to uniocular poor vision (logMAR 0.9) from previous retinal detachment. This left a total of 35 patients, the demographics of whom are shown in Table 1.

At presentation, $17(49 \%)$ patients used monocular occlusion to avoid diplopia; the remainder used a prism and/ or abnormal head posture. In $28(80 \%)$ patients it was possible to achieve fusion of images during motility examination; in the remainder fusion was not possible due to excyclotorsion. In the 28 patients where fusion was possible, mean (std) vertical fusion range was $7.0 \Delta(4.2 \Delta)$, range $0-15 \Delta$. A normal fusion range $(<5 \Delta)$ was the case for $9(32.1 \%)$, whilst $7(25 \%)$ had a moderately increased

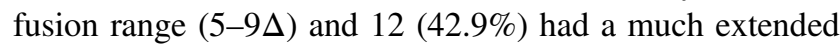
fusion range $(\geq 10 \Delta)$.

A disparity between vertical deviations in primary position and downgaze was common; median (IQR) vertical disparity was $4 \Delta(2-10 \Delta)$ with $9(25.7 \%)$ patients having a $10 \Delta$ or more difference between vertical deviation measured at distance in primary position when compared with downgaze.

Based on our surgical principles, a total of $6(17.1 \%)$ patients had four muscles recessed during the first surgery, 13 (37.1\%) had three muscles recessed, 10 (28.6\%) had two muscles recessed and $6(17.1 \%)$ had just one muscle recession. In terms of vertical muscles; 9 (25.7\%) patients had one vertical muscle recessed (six unilateral inferior rectus recession and three unilateral superior rectus recession); 16 (45.7\%) had two vertical muscles recessed (six bilateral inferior recti recessions, ten contralateral inferior rectus/superior rectus 
Table 2 Prism cover test and duction deficit examination findings pre and 3 months post single surgery for the 35 patients

\begin{tabular}{lccc}
\hline Prism cover tests & Preoperatively & 3 months postoperatively & $\begin{array}{c}p \text {-value (Wilcoxon } \\
\text { signed-rank test) }\end{array}$ \\
\hline Angle of vertical deviation in PP, median (IQR) & $20 \Delta(15,30 \Delta)$ & $1 \Delta(0,3 \Delta)$ & $<0.001^{*}$ \\
Angle of vertical deviation in downgaze, median (IQR) & $15 \Delta(8,22 \Delta)$ & $1 \Delta(0,3 \Delta)$ & $<0.001^{*}$ \\
Angle of horizontal deviation in PP, median (IQR) & $7 \Delta(2,16 \Delta)$ & $2 \Delta(0,4 \Delta)$ & $<0.001^{*}$ \\
Angle of horizontal deviation in downgaze, median (IQR) & $6 \Delta(1.8,12.5 \Delta)$ & $1 \Delta(0-2 \Delta)$ & $<0.001^{*}$ \\
Mean ductions & & $0(0,0)$ & 0.275 \\
Hypertropic eye downgaze duction, median (IQR) & $0(-0.5,0)$ & $0(0,0)$ & 0.054 \\
Hypotropic eye downgaze duction, median (IQR) & $0(0,0)$ & $-1(-1.5,0)$ & 0.146 \\
Hypertropic eye upgaze duction, median (IQR) & $-1(-2.5,-1)$ & $-1(-2,-0.5)$ & $<0.001^{*}$ \\
Hypotropic eye upgaze duction, median (IQR) & $-3(-4,-2.5)$ & \\
\hline
\end{tabular}

$\Delta$ prism dioptres, $P P$ primary position, $I Q R$ inter-quartile range

recessions); and 10 (28.6\%) had three muscle surgery (eight bilateral inferior rectus recessions with unilateral superior rectus recession; two bilateral superior rectus recessions with unilateral inferior rectus recession).

We used an adjustable suture in $9(26 \%)$ patients, of these eight were on a superior rectus and one on a lateral rectus. The majority $(66 \%)$ of these were tied down without adjustment; with two being adjusted by $0.5-1 \mathrm{~mm}$ (both superior recti, one advancement, one recession); and one recessed a further 3-4 $\mathrm{mm}$ (superior rectus). The patient requiring this significant adjustment had had a vertical deviation of $75 \Delta$ in primary position and $50 \Delta$ in downgaze preoperatively.

Table 2 shows the pre- and postoperative measurements for prism cover tests and ductions. The median sizes of vertical deviations in primary position and downgaze preoperatively and after a single surgery are shown in Fig. 1.

After a single surgery, $29(82.9 \%)$ patients had functional BSV without a prism in primary position. A further one patient achieved this with a $2 \Delta$ base out prism giving a total of $(85.7 \%)$ patients with BSV in primary position with no or minimal prismatic correction. The same 29 (82.9\%) patients had BSV in primary position and in functional downgaze and $28(80 \%)$ patients had BSV in functional upgaze. Four patients $(11 \%)$ had a reversal of their vertical deviation in functional downgaze and all of these patients went on to have further surgery.

Disparity between vertical deviation in primary position and downgaze reduced postoperatively to a median (IQR) of $1 \Delta(0-2 \Delta)$; with only one patient having a disparity of $10 \Delta$ or greater (a patient with reactivation of her TED). All other patients had a disparity of $5 \Delta$ or less and 30 patients $(86 \%)$ had disparity of $2 \Delta$ or less.

Second surgery was required in $5(14 \%)$ patients; these are the five outliers in Fig. 1 for postoperative measurements in primary position (the additional outlier for downgaze measurement had a postoperative phoria of $8 \mathrm{D}$ in downgaze, which she was able to control with her extended

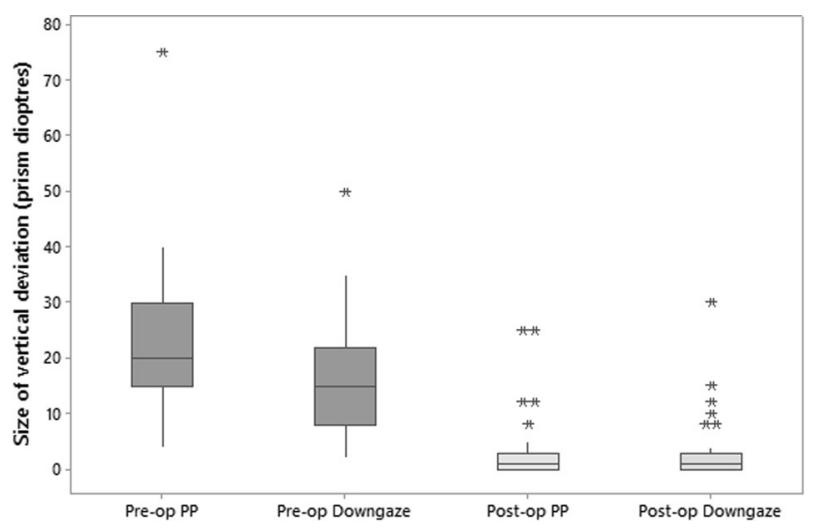

Fig. 1 A box and whisker plot which shows the median and interquartile range for vertical deviation in prism dioptres. This is shown in primary position (PP) and downgaze both pre and after a single surgery. Stars represent outliers

fusional reserve). For the five patients requiring second surgery, in three this was anticipated because of the complex nature of their strabismus. In one patient there was an undercorrection with a residual vertical tropia. And one patient had reactivation of TED, which required subsequent bilateral decompressions and a third strabismus surgery. Two patients requiring re-operation had a disparity between primary and downgaze preoperatively of $10 \Delta$ or more, one of these patients had a residual vertical deviation and one had a reversal of their deviation.

Median follow-up time was 16 months, or 467 days (IQR 277-797). At final follow-up, 32 (91\%) were diplopia free without prisms with BSV in both primary position and functional downgaze; $3(9 \%)$ used a prism correction $(2 \Delta$ in two patients and $12 \Delta$ in the one patient who had had three surgeries).

An example of a patient with vertical incomitance and an enlarged vertical fusion range is shown in Fig. 2. An example of a patient with vertical incomitance without an enlarged vertical fusion range is shown in Fig. 3. 

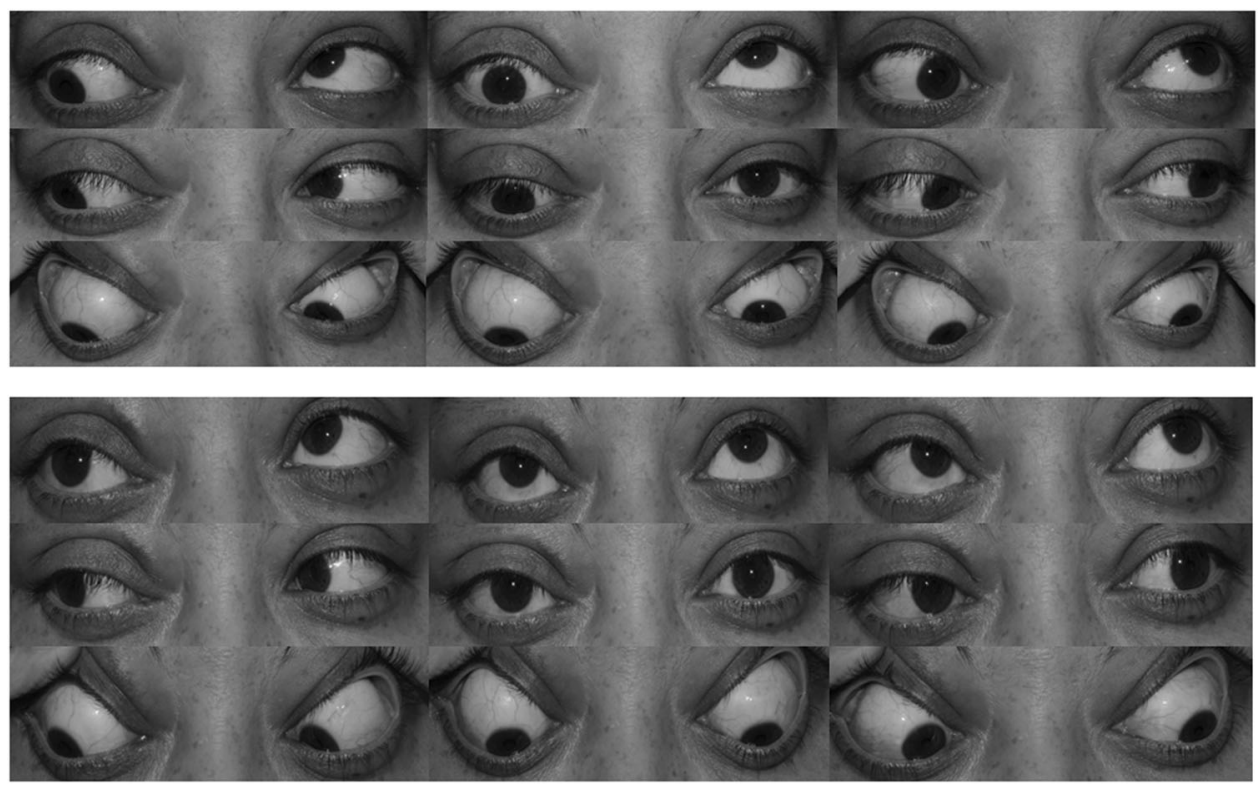

Fig. 2 This patient had a right hypotropia, which measured $40 \Delta$ in primary position and $25 \Delta$ in downgaze. She had a small $(8 \Delta)$ exotropia in primary position. She had an enlarged vertical prism fusion range of $15 \Delta$ in the direction of vertical misalignment. She had asymmetry of her duction deficits with -5 restriction of right upgaze and full left upgaze. Perioperative FDTs showed -6 restriction of right elevation, but no restriction of left elevation and normal downgaze ductions. A target angle of correction of $<30$ was chosen, in the

\section{Discussion}

This study shows the results of strabismus surgery when prioritising downgaze alignment for patients with vertical strabismus as a consequence of TED. The 35 patients included represent a typical cohort for TED, with $46 \%$ of patients having had previous decompression surgery, 29\% having previous orbital radiotherapy, and 25\% having angles of vertical deviations in excess of $30 \Delta$ in primary position. All patients had preoperative diplopia and almost $50 \%$ of patients required monocular occlusion preoperatively for symptom relief. The vertical strabismus was often highly incomitant with vertical disparities between primary position and downgaze of more than $10 \Delta$ in $26 \%$ patients. Nevertheless, surgical success rate was high, with 83\% patients achieving BSV in primary position and functional downgaze without prismatic correction after a single surgery. We found $86 \%$ patients required no, or minimal $(<3 \Delta)$ prismatic correction at 3 months postoperatively and we report a re-operation rate of $14 \%$. At final follow-up, $97 \%$ patients required no or minimal $(<3 \Delta)$ prismatic correction.

In comparison with other, previously published algorithms, we place a greater emphasis on the angle of deviation in downgaze and in doing so avoid an early/medium term reversal of vertical strabismus in downgaze which we knowledge she could use her vertical and horizontal motor fusion reserve in primary position. She had a right inferior recession of $5.5 \mathrm{~mm}$ and a left superior rectus recession of $3.5 \mathrm{~mm}(9 \mathrm{~mm}$ total) Postoperatively she was orthophoric in primary position and downgaze; with BSV in primary position, extreme downgaze, and to $15^{\circ}$ upgaze. She had full and symmetrical downgaze ductions, with reduced but still restricted upgaze ductions ( -3 right and -1 left)

know is poorly tolerated [17]. Our results compare favourably to previously published series using a variety of algorithms and techniques (see Table 3).

We have found it helpful to exploit the patient's potential pre-existing vertical fusion amplitudes. This enables us to surgically correct the vertical deviation in functional downgaze while relying on vertical fusion to maximise the field of BSV towards (and in) primary position by reducing the "manifest" vertical disparity between downgaze and primary position. Therefore, for patients where the vertical fusion range is extended relative to the disparity, we can allow more undercorrection of the vertical deviation. Where there is any manifest undercorrection in primary position, patients can use a small chin-up position to compensate in the immediate postoperative period and await the effects of muscle sequelae creating a new equilibrium in fusing patients. We know that in non-restrictive vertical strabismus that these changes can continue for 12 months or more [19].

The incomitance which we commonly saw preoperatively between primary position and downgaze can be considered as an additional reflection of the mechanical imbalance between opposing muscle groups. This incomitance is a particular challenge in managing patients with strabismus in TED. Our surgical principles aimed to achieve downgaze duction symmetry, which enabled us to achieve 

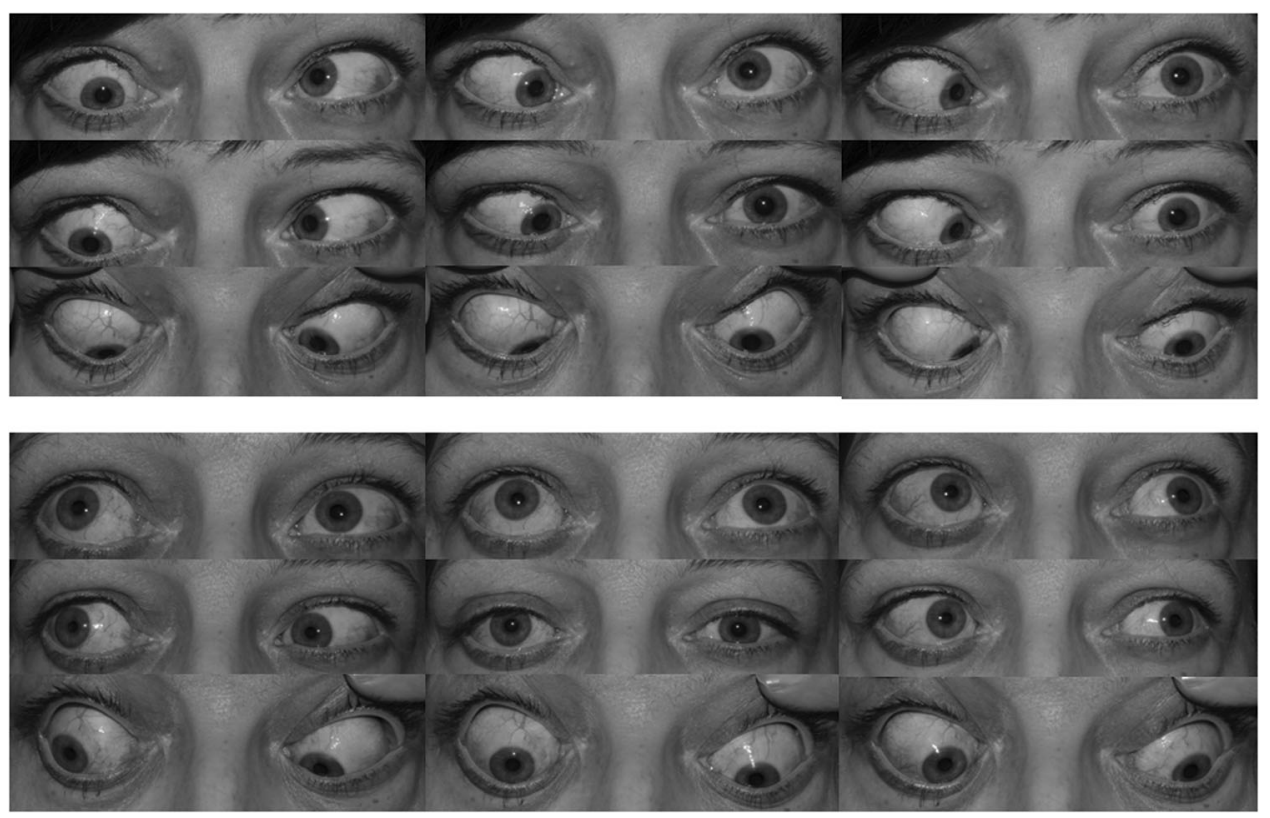

Fig. 3 This patient had a right hypotropia, which measured $30 \Delta$ in primary position and $15 \Delta$ in extreme downgaze, as well as a marked esotropia measuring $60 \Delta$ in primary position. It was difficult to achieve fusion preoperatively but she could achieve tenuous BSV in free space, in downgaze with prismatic correction. She had a prism fusion range of $5 \Delta$ in the direction of vertical misalignment (able to achieve tenuous BSV in downgaze with a $40 \Delta$ base out prism and from 15 to $20 \Delta$ base up right eye). She had asymmetrical, restricted ductions as shown. Perioperative FDTs on the right showed restrictions of the order -6 to elevation, -1 to depression, -5 to abduction

an equilibrium between the opposing restrictive and innervational forces, substantially reducing the vertical disparity between primary position and downgaze postoperatively. We feel that this is one of the reasons for our patient group continuing to maintain excellent alignment at a median of 16 months follow-up. Furthermore, by prioritising downgaze alignment and utilising any fusional reserves we were able to achieve good surgical outcomes even for those with significant disparity between primary position and downgaze preoperatively (see Figs. 2 and 3 for examples of how this disparity was managed with and without enlarged fusional reserves respectively). Note that the area of BSV achieved for the patient with an extended fusion range (Fig. 2) was greater than that of the patient with a normal fusion range (Fig. 3). Note also that the extended fusion range (Fig. 2) enabled us to allow more undercorrection of the vertical deviation.

We do not routinely use adjustable sutures, and where we have these have been placed on the superior rectus as this is often less affected by thyroid strabismus and hence may be more accurately manipulated in the awake patient. Furthermore, superior rectus adjustment is less prone to significantly modifying downgaze duction symmetry that can and -2 to adduction. FDTs on the left showed -3 to depression, -2.5 to elevation, -3.5 to abduction and free to adduction. A target angle of vertical correction was taken as 20-25 dioptres, the right inferior rectus was recessed $8.5 \mathrm{~mm}$, leaving it at $15 \mathrm{~mm}$ from the limbus. She also had a net total of $11.5 \mathrm{~mm}$ of horizontal surgery with bimedial rectus recessions. Postoperatively she had a small vertical phoria in primary position (two dioptres right hypophoria) with reversal of her hyoptropia only in extreme downgaze (four dioptres left hypotropia). She had BSV in primary position, up to $20^{\circ}$ of upgaze and extending $>40^{\circ}$ in downgaze

be associated with the sequelae of inferior rectus muscle adjustment, where late progressive postadjustment overcorrection remains a well-recognised complication [20-22]. A minority of the adjustable sutures we used needed adjustment, with one noteworthy patient who had a $75 \Delta$ vertical deviation in primary position.

Barker et al. [16] recently suggested aiming for $8 \Delta$ of undercorrection for thyroid vertical strabismus, to avoid postoperative drift. Volpe et al., also anticipate a drift towards overcorrection, but almost a quarter of their patients ended up undercorrected [13]. In our study, we have not witnessed this tendency to overcorrection, despite our median follow-up of 16 months. However, as we chose a target angle of correction for the functional downgaze deviation, this does result in an effective undercorrection of the primary position deviation in those patients with significant disparity between primary position and downgaze deviations. Indeed, the mean disparity of vertical deviation in our study of $6.4 \Delta$ is not far off the $8 \Delta$ undercorrection suggested by Barker et al [16]. Like Kim et al., we use reasonably standard dosing for thyroid strabismus (assuming that $1 \mathrm{~mm}$ of surgery gives 2.5-3 $\Delta$ correction) [8]. We think that this method works with our 


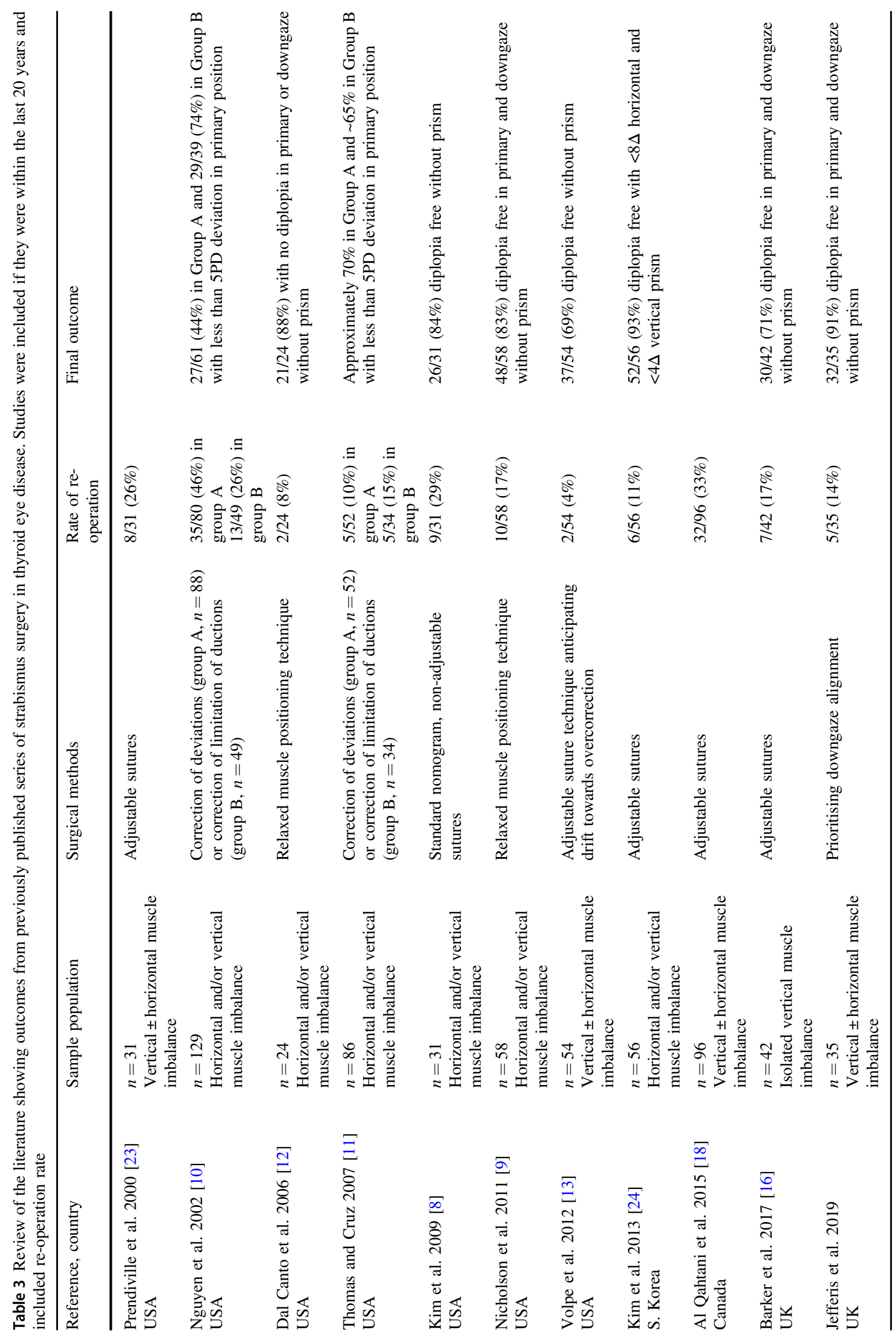


surgical principles because of the emphasis that we place on relieving restrictions whilst adhering to downgaze duction symmetry. By relieving and symmetrising ductions we attempt to alleviate those factors that make strabismus surgery unpredictable in TED. This is analogous to methods that concentrate on release of duction deficits $[9,10]$, and in line with others who have found that surgery tailored to relieve restrictive ductions of opposing recti aids success [23]. Our approach was also successful at avoiding postoperative complications, such as postoperative A-patterns and new torsional strabismus. In patients where excyclotorsion was a barrier to achieving BSV preoperatively, when the excyclotorsion effect of the inferior recti was eliminated (by recession) the torsional element of the strabismus was invariably relieved.

While our study reports a high level of success using our methods, we concede that there are limitations to this report. The study is retrospective and relates to a single surgeon in a single centre, it is not given that these results will be replicated by other surgeons in other units [11]. However, we think that the principles described are simple to use and adapt and may well be applicable in a wide range of settings.

In conclusion, our study describes highly favourable outcomes for patients with vertical strabismus and TED using a series of surgical principles combined with standard surgical dosing. The principles place emphasis on magnitude of deviation in functional downgaze, utilising any preexisting enlarged vertical fusion amplitudes, and seeking to symmetrise downgaze ductions. In this way, reversal of diplopia in downgaze can be avoided.

\section{Summary}

\section{What was known before?}

- Thyroid eye disease (TED) can cause a restrictive, incomitant, vertical strabismus whose spectrum represents a challenge to the strabismus surgeon; this is reflected in the unforeseen outcomes and high reoperation rates described in the literature.

- Standard surgical dosing is generally considered inadequate alone when planning surgery.

- Other methods such as the use of adjustable sutures, correction of duction deficits and intraoperative relaxed muscle techniques are utilised with varying success.

\section{What this study adds?}

- We describe our approach to managing vertical strabismus in TED that is novel, in that it prioritises alignment in downgaze and exploits any pre-existing extended vertical fusional reserves.

- A substantial minority of TED patients (some 26\%, 9/35 in this study) can be expected to have a therapeutic/ surgically significant vertical misalignment disparity of $>10$ prism dioptres between primary position and downgaze preoperatively. This can be effectively managed using the surgical principles we describe.

- In our series $83 \%$ patients achieved the desired outcome of binocular single vision in downgaze and primary position after a single surgery. Additional strabismus surgery was required in $14 \%$ and $91 \%$ were diplopia free without prisms at a median of 16 months follow-up.

\section{Compliance with ethical standards}

Conflict of interest The authors declare that they have no conflict of interest.

Publisher's note Springer Nature remains neutral with regard to jurisdictional claims in published maps and institutional affiliations.

\section{References}

1. Scott WE, Thalacker JA. Diagnosis and treatment of thyroid myopathy. Ophthalmology. 1981;88:493-8.

2. Calcutt C. Dysthyroid eye disease from the inside looking out. Aust Orthopt J. 1994;30:67-70.

3. Harcourt B. Ocular motility disorders associated with thyroid disease. Br Orthopt J. 1982;39:1-6.

4. Wallang B, Kekunnaya R, Granet D. Strabismus surgery in thyroid-related eye disease: strategic decision making. Curr Ophthalmol Rep. 2015;1:218-28.

5. Dagi LR. Management of graves myopathy: understanding and managing vertical strabismus from thyroid eye disease. J AAPOS. 2018;22:252-5.

6. Dagi LR, Elliott AT, Roper-Hall G, Cruz OA. Thyroid eye disease: honing your skills to improve outcomes. J AAPOS. 2010;14:425-31.

7. Harrad R. Management of strabismus in thyroid eye disease. Eye $2015 ; 29: 234-7$.

8. Kim SH, Rotberg L, Sprunger DT. Standard strabismus surgery in thyroid ophthalmopathy. Binocul Vis strabismus Q. 2009;24:86-92.

9. Nicholson BP, De Alba M, Perry JD, Traboulsi EI. Efficacy of the intraoperative relaxed muscle positioning technique in thyroid eye disease and analysis of cases requiring reoperation. J AAPOS. 2011;15:321-5.

10. Nguyen VT, Park DJ, Levin L, Feldon SE. Correction of restricted extraocular muscle motility in surgical management of strabismus in graves' ophthalmopathy. Ophthalmology 2002;109:384-8.

11. Thomas SM, Cruz OA. Comparison of two different surgical techniques for the treatment of strabismus in dysthyroid ophthalmopathy. J AAPOS. 2007;11:258-61.

12. Dal Canto AJ, Crowe S, Perry JD, Traboulsi EI. Intraoperative relaxed muscle positioning technique for strabismus repair in thyroid eye disease. Ophthalmology 2006;113:2324-30. 
13. Volpe NJ, Mirza-George N, Binenbaum G. Surgical management of vertical ocular misalignment in thyroid eye disease using an adjustable suture technique. J Aapos 2012;16:518-22.

14. Lueder GT, Scott WE, Kutschke PJ, Keech RV. Long-term results of adjustable suture surgery for strabismus secondary to thyroid ophthalmopathy. Ophthalmology 1992;99:993-7.

15. Gardner TA, Kennerdell JS. Treatment of dysthyroid myopathy with adjustable suture recession. Ophthalmic Surg 1990;21: 519-21.

16. Barker L, Mackenzie K, Adams GG, Hancox J. Long-term surgical outcomes for vertical deviations in thyroid eye disease. Strabismus 2017;25:67-72.

17. Flanders M, Hastings M. Diagnosis and surgical management of strabismus associated with thyroid-related orbitopathy. J Pediatri Ophthalmol strabismus 1997;34:333-40.

18. Al Qahtani ES, Rootman J, Kersey J, Godoy F, Lyons CJ. Clinical pearls and management recommendations for strabismus due to thyroid orbitopathy. Middle East Afr J Ophthalmol 2015;22: 307-11.
19. Raoof N, Burke JP. Isolated inferior oblique myectomy for vertical deviations of at least 20 prism diopters in the primary position. J Aapos 2016;20:112-6.

20. Cestari DM, Freire MV, Chun BY. Vertical rectus muscle recession versus combined vertical and horizontal rectus muscle recession in patients with thyroid eye disease and hypotropia. $\mathbf{J}$ Aapos 2018;22:257-61.

21. Sprunger DT, Helveston EM. Progressive overcorrection after inferior rectus recession. J Pediatr Ophthalmol Strabismus 1993;30:145-8.

22. Peragallo JH, Velez FG, Demer JL, Pineles SL. Postoperative drift in patients with thyroid ophthalmopathy undergoing unilateral inferior rectus muscle recession. Strabismus 2013;21:23-28.

23. Prendiville P, Chopra M, Gauderman WJ, Feldon SE. The role of restricted motility in determining outcomes for vertical strabismus surgery in Graves' ophthalmology. Ophthalmology 2000;107:545-9.

24. Kim MH, Park KA, Oh SY. The effect of previous orbital decompression on results of strabismus surgery in patients with Graves' ophthalmopathy. J AAPOS. 2013;17:188-191. 21 